\title{
A Few Remarks on Robust Estimation of Power Spectra
}

\author{
Georgy Shevlyakov Nickolay Lyubomishchenko Pavel Smirnov \\ St. Petersburg State Polytechnic University
}

\begin{abstract}
Various robust versions of the classical methods of power spectra estimation are considered. Their performance evaluation is studied in autoregressive models with contamination. It is found out that the best robust estimates of power spectra are based on robust highly efficient estimates of autocovariances. Several open problems for future research are formulated.
\end{abstract}

Keywords: power spectra, robustness, autoregressive models.

\section{A Few Remarks on Robust Estimation of Power Spectra}

\author{
Georgy Shevlyakov, Nickolay Lyubomishchenko and Pavel Smirnov \\ St. Petersburg State Polytechnic University, Russia
}

\begin{abstract}
Various robust versions of the classical methods of power spectra estimation are considered. Their performance evaluation is studied in autoregressive models with contamination. It is found out that the best robust estimates of power spectra are based on robust highly efficient estimates of autocovariances. Several open problems for future research are formulated.
\end{abstract}

Keywords: power spectra, robustness, autoregressive models.

\section{Introduction}

Robust methods ensure high stability of statistical inference under uncontrolled deviations from the assumed distribution model. Much less attention is devoted in the literature to robust estimation of data spectra as compared to robust estimation of location, scale, regression and covariance (Huber, 1981; Hampel, Ronchetti, Rousseeuw, and Stahel, 1986; Maronna, Martin, and Yohai, 2006). However, it is necessary to study these problems due to their both theoretical and practical importance (estimation of time series power spectra in various applications, such as communication, geophysics, medicine, etc.), and also because of the instability of classical methods of power spectra estimation in the presence of outliers in the data (Kleiner, Martin, and Thomson, 1979). 
There are several classical approaches to estimation of the power spectra of time series, e.g., via the nonparametric periodogram and the Blackman-Tukey formula methods, as well as via the parametric Yule-Walker and filter-based methods (Blackman and Tukey, 1958; Bloomfield, 1976; Brockwell and Davis, 1991). Thereafter, we may consider their various robust versions: to the best of our knowledge, a first systematic study of them is made in the dissertation of Bernhard Spangl (Spangl, 2008).

In what follows, we partially use the aforementioned study as a baseline, mostly follow the classification of robust methods of power spectra estimation given in (Spangl, 2008), specify them and propose some new approaches with their comparative performance evaluation. Basically, to obtain good robust estimates of power spectra, we use highly efficient robust estimates of scale and correlation (Shevlyakov and Smirnov, 2011).

Our main goals are both to outline the existing approaches to robust estimation of power spectra and to indicate open problems, so our paper is partially a review and partially a program for a future research.

The remainder of the paper is as follows. In Section 2, classical methods of power spectra estimation are briefly enlisted. In Section 3, robust modifications of classical approaches are formulated. In Section 4, a few preliminary results on the comparative study of the performance evaluation of various robust methods are represented. In Section 5, some conclusions and open problems for future research are drawn.

\section{Classical estimation of power spectra}

\subsection{Nonparametric estimation of power spectra}

The nonparametric approach to estimation of power spectra is based on smoothed periodograms (Blackman and Tukey, 1958; Bloomfield, 1976).

Let $x_{t}, t=1, \ldots, n$ be a second-order stationary time-series with zero mean. Assume that the time intervals between two consecutive observations are equally spaced with duration $\Delta t$. Then the periodogram is defined as follows:

$$
\widehat{S}_{P}(f)=\Delta t / n\left|\sum_{t=1}^{n} x_{t} \exp \{-i 2 \pi f t \Delta t\}\right|^{2}
$$

over the interval $\left[-f_{(n)}, f_{(n)}\right]$, where $f_{(n)}$ is the Nyquist frequency: $f_{(n)}=1 /(2 \Delta t)$.

The Blackman-Tukey formula gives the representation of formula (1) via the sample autocovariances $\hat{c}_{x x}$ of the time series $x_{t}$ (Blackman and Tukey, 1958):

$$
\widehat{S}_{P}(f)=\widehat{S}_{B T}(f)=\Delta t \sum_{h=-(n-1)}^{n-1} \hat{c}_{x x}(h) \exp \{-i 2 \pi f h \Delta t\} .
$$

It can be seen that the periodogram $\widehat{S}_{P}(f)(1)$ at the frequency $f=f_{k}=k /(n \Delta t)$, where $k$ is an integer such that $k \leq\lfloor n / 2\rfloor$, is equal to the squared absolute value of the discrete Fourier transform $X\left(f_{k}\right)$ of the sequence $x_{1}, \ldots, x_{n}$ given by the following formula

$$
X\left(f_{k}\right)=\Delta t \sum_{t=1}^{n} x_{t} \exp \left\{-i 2 \pi f_{k} t \Delta t\right\}
$$

To reduce the bias and variance of the periodogram $\widehat{S}_{P}(f)$, the conventional techniques based on tapering and averaging of periodograms is used (Bloomfield, 1976). 


\subsection{Parametric estimation of power spectra}

The widely used form of a parametric power spectra estimation procedure exploits an autoregressive model of order $p$ for the underlying power spectrum $S(f)$. A stationary $A R(p)$ process $x_{t}$ with zero mean is described by the following equation

$$
x_{t}=\sum_{j=1}^{p} \phi_{j} x_{t-j}+\epsilon_{t}
$$

where $\epsilon_{t}$ are i.i.d. Gaussian white noises with zero mean and variance $\sigma_{\epsilon}^{2}$. The power spectrum estimate $\widehat{S}_{A R}(f)$ has the form (Bloomfield, 1976)

$$
\widehat{S}_{A R}(f)=\frac{\Delta t \hat{\sigma}_{\epsilon}^{2}}{\left|1-\sum_{j=1}^{p} \hat{\phi}_{j} \exp \{-i 2 \pi f j \Delta t\}\right|^{2}}, \quad|f| \leq f_{(n)},
$$

where $\hat{\phi}_{1}, \ldots, \hat{\phi}_{p}$ and $\hat{\sigma}_{\epsilon}^{2}$ are the maximum likelihood estimates of the model parameters.

\section{Robust estimation of power spectra}

\subsection{Preliminaries}

A natural way to provide robustness of the classical estimates of power spectra is based on using highly robust and efficient estimates of location, scale and correlation in the classical estimates. Here we enlist several highly robust and efficient estimates of scale and correlation. Robust Scale: The median absolute deviation $M A D_{n}(x)=\operatorname{med}|x-\operatorname{med} x|$ is a highly robust estimate of scale with the maximal value of the breakdown point 0.5 , but its efficiency is only 0.37 at the normal distribution (Hampel, Ronchetti, Rousseeuw, and Stahel, 1986). In (Rousseeuw and Croux, 1993), a highly efficient robust estimate of scale $Q_{n}$ has been proposed: it is close to the lower quartile of the absolute pairwise differences $\left|x_{i}-x_{j}\right|$, and it has the maximal breakdown point 0.5 as for $M A D_{n}$ but much higher efficiency 0.82 . The drawback of this estimate is its low computation speed; the computation of $Q_{n}$ requires an order of greater time than of $M A D_{n}$.

In (Smirnov and Shevlyakov, 2010), an $M$-estimate of scale denoted by $F Q_{n}$ whose influence function is approximately equal to the influence function of the estimate $Q_{n}$ is proposed

$$
\begin{gathered}
F Q_{n}(x)=1.483 \operatorname{MAD}(x)\left(1-\left(Z_{0}-n / \sqrt{2}\right) / Z_{2}\right) \\
Z_{k}=\sum_{i=1}^{n} u_{i}^{k} e^{-u_{i}^{2} / 2}, \quad u_{i}=\left(x_{i}-\operatorname{med} x\right) /\left(1.483 M A D_{n}\right), k=0,2 ; \quad i=1, \ldots, n .
\end{gathered}
$$

The efficiency and breakdown point of $F Q_{n}$ are equal to 0.81 and to 0.5 , respectively.

Robust Correlation: A remarkable robust minimax bias and variance $M A D$ correlation coefficient with the breakdown point 0.5 and efficiency 0.37 is given by

$$
r_{M A D}(x, y)=\left(M A D^{2}(u)-M A D^{2}(v)\right) /\left(M A D^{2}(u)+M A D^{2}(v)\right),
$$

where $u$ and $v$ are the robust principal variables (Shevlyakov and Smirnov, 2011)

$$
u=\frac{x-\operatorname{med} x}{\sqrt{2} M A D x}+\frac{y-\operatorname{med} y}{\sqrt{2} M A D y}, \quad v=\frac{x-\operatorname{med} x}{\sqrt{2} M A D x}-\frac{y-\text { med } y}{\sqrt{2} M A D y} .
$$

Much higher efficiency 0.81 with the same breakdown point 0.5 can be provided by using the FQ correlation coefficient (Shevlyakov and Smirnov, 2011)

$$
r_{F Q}(x, y)=\left(F Q^{2}(u)-F Q^{2}(v)\right) /\left(F Q^{2}(u)+F Q^{2}(v)\right) .
$$




\subsection{Robust $L_{p}$-norm analogs of the discrete Fourier transform}

Since computation of the discrete Fourier transform (DFT) (3) is the first step in periodogram estimation of power spectra, consider the following robust $L_{p}$-norm analogs of the DFT.

As the classical DFT (3) $X(f)$ can be obtained via the $L_{2}$-norm approximation to the data $y_{t}(f)=x_{t} \exp \{-i 2 \pi f t \Delta t\}, t=1, \ldots, n$ :

$$
X(f) \propto \arg \min _{Z} \sum_{t=1}^{n}\left|y_{t}(f)-Z\right|^{2},
$$

the $L_{p}$-norm analog of $X(f)$ (up to the scale factor) is defined as follows :

$$
X_{L_{p}}(f) \propto \arg \min _{Z}\left\{\sum_{t=1}^{n}\left|y_{t}(f)-Z\right|^{p}\right\}^{1 / p}, \quad 1 \leq p<\infty .
$$

The case of $1 \leq p<2$, and especially the $L_{1}$-norm or the median Fourier transform, are of our particular interest (Pashkevich and Shevlyakov, 1995; Spangl and Dutter, 2005; Spangl, 2008):

$$
X_{L_{1}}(f) \propto \arg \min _{Z}\left\{\sum_{t=1}^{n}\left|y_{t}(f)-Z\right|\right\} .
$$

The other possibilities such as the component-wise, spatial medians, and trimmed mean analogs of the DFT are also considered in (Pashkevich and Shevlyakov, 1995; Spangl, 2008).

\subsection{Robust nonparametric estimation}

Now we apply the aforementioned robust analogs of the DFT as well as highly robust and efficient estimates of scale and correlation to the classical nonparametric estimation of power spectra.

Robust Nonparametric Estimation via Periodograms: Here we apply the robust $L_{p}$-norm analogs of the DFT to the classical periodogram $\widehat{S}_{P}(f)(1)$ :

$$
\widehat{S}_{L_{p}}(f) \propto\left|X_{L_{p}}(f)\right|^{2}
$$

In what follows, the $L_{1}$ - or the median periodogram is of our particular interest.

Robust Nonparametric Estimation via the Blackman-Tukey Formula: In order to construct robust modifications of the Blackman-Tukey formula, we have to consider robust estimates of autocovariances $\widehat{c}_{x x}(h)$ instead of the conventional ones used in (2). These robust estimates are based on the highly robust $M A D$ and $F Q$ estimates of scale and correlation (6) - (8):

$$
\begin{gathered}
\widehat{c}_{M A D}(h)=r_{M A D}\left(x_{t}, x_{t-h}\right) M A D\left(x_{t}\right) M A D\left(x_{t-h}\right)=r_{M A D}(h) M A D^{2}(x), \\
\widehat{c}_{F Q}(h)=r_{F Q}\left(x_{t}, x_{t-h}\right) F Q\left(x_{t}\right) F Q\left(x_{t-h}\right)=r_{F Q}(h) F Q^{2}(x) .
\end{gathered}
$$

To provide the required Teplitz property (symmetry, semipositive definiteness, equal elements on sub-diagonals) of the autocovariance matrix $\widehat{C}_{x x}$ built of the element-wise robust autocovariances (12), a new effective transform is used (Letac, 2011). Thus, the Teplitz transformed estimates are substituted into formula $(2)$, and the corresponding robust estimates of power spectra are denoted as $\widehat{S}_{M A D}(f)$ and $\widehat{S}_{F Q}(f)$, respectively.

\subsection{Robust parametric estimation of power spectra via the Yule-Walker equations}

A classical approach to estimation of autoregressive parameters $\phi_{1}, \ldots, \phi_{p}$ in (4) is based on 
the solution of the linear system of the Yule-Walker equations (Bloomfield, 1976):

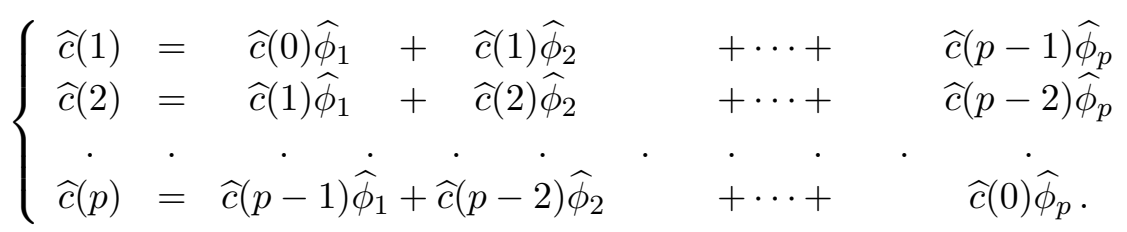

The estimate of the innovation noise variance is defined by the following equation

$$
\widehat{c}(0)=\widehat{c}(1) \widehat{\phi}_{1}+\widehat{c}(2) \widehat{\phi}_{2}+\cdots+\widehat{c}(p) \widehat{\phi}_{p}+\widehat{\sigma}_{\epsilon}^{2} .
$$

Substituting robust estimates of autocovariances (12) into (13) and (14), we get the robust analogs of the Yule-Walker equations. Solving these equations, we arrive at the robust estimate of power spectra in the form (5).

\subsection{Robust parametric estimation via filtering}

A wide collection of robust methods of power spectra estimation is given by various robust filters (Kalman, Masreliez, ACM-type, robust least squares, filter-cleaners, etc.) providing preliminary cleaning the data with the subsequent power spectra estimation. An extended comparative experimental study of robust filters is made in (Spangl and Dutter, 2005; Spangl, 2008); below we compare some of those results with ours.

\section{Performance evaluation}

\subsection{Robustness of the median Fourier transform power spectra}

The median Fourier transform power spectra estimate $\widehat{S}_{L_{1}}(f) \propto\left|X_{L_{1}}(f)\right|^{2}$ inherits the maximum value of the sample median breakdown point $\varepsilon^{*}=1 / 2$.

Theorem The breakdown point of $\widehat{S}_{L_{1}}(f)$ is equal to $1 / 2$. Here, the breakdown point $\varepsilon^{*}$ is understood as the maximal ratio of the number of unbounded observations in the data sample under which the estimate still remains bounded (Hampel, Ronchetti, Rousseeuw, and Stahel, 1986).

Fig. 1 illustrates this phenomenon: the observed realisation is the mixture of $\sin (\pi t / 4)$ and $\sin (\pi t / 8)$ on the $40 \%$ and on the $60 \%$ of the interval of observation, respectively. In this case, the classical periodogram indicates the presence of both peaks whereas the median periodogram indicates only one spectrum peak, which corresponds to the dominating signal $\sin (\pi t / 8)$.

\subsection{Additive outlier contamination model}

In Monte Carlo experiment, an autoregressive model is used because of, first, it is a direct stochastic counterpart of an ordinary differential equation, second, an autoregressive model is the maximum entropy parametric approximation to an arbitrary strictly stationary random process (Cover and Thomas, 1991).

In this paper, we use the autoregressive models $\mathrm{AR}(2): x_{t}=x_{t-1}-0.9 x_{t-2}+\epsilon_{t}$ and $\mathrm{AR}(4)$ : $x_{t}=x_{t-1}-0.9 x_{t-2}+0.5 x_{t-3}-0.1 x_{t-4}+\epsilon_{t}$ together with Gaussian additive outliers (AO) with pdf $N(x ; 0,10)$. The comparative study is performed on different sample sizes $n$ and numbers of trials $M$ (see, Figs. 2-4).

\subsection{Disorder contamination model}

In this paper, we propose a contamination model dubbed as a disorder contamination describing the violations of the thin structure of a random process, when an $A R$-process is shortly 
Mixture process realisation
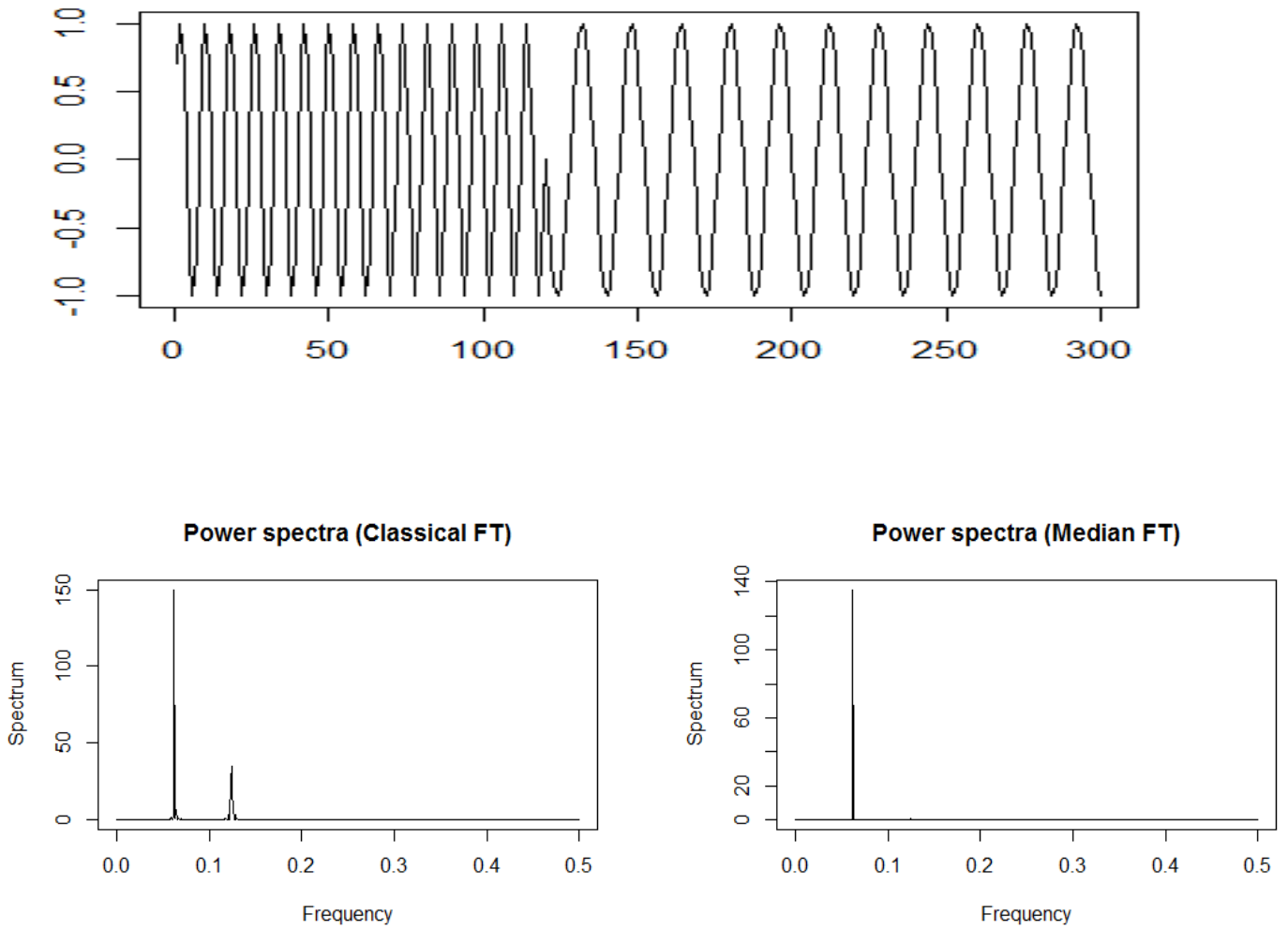

Figure 1: Median Fourier transform breakdown point $\varepsilon^{*}=0.5$ property

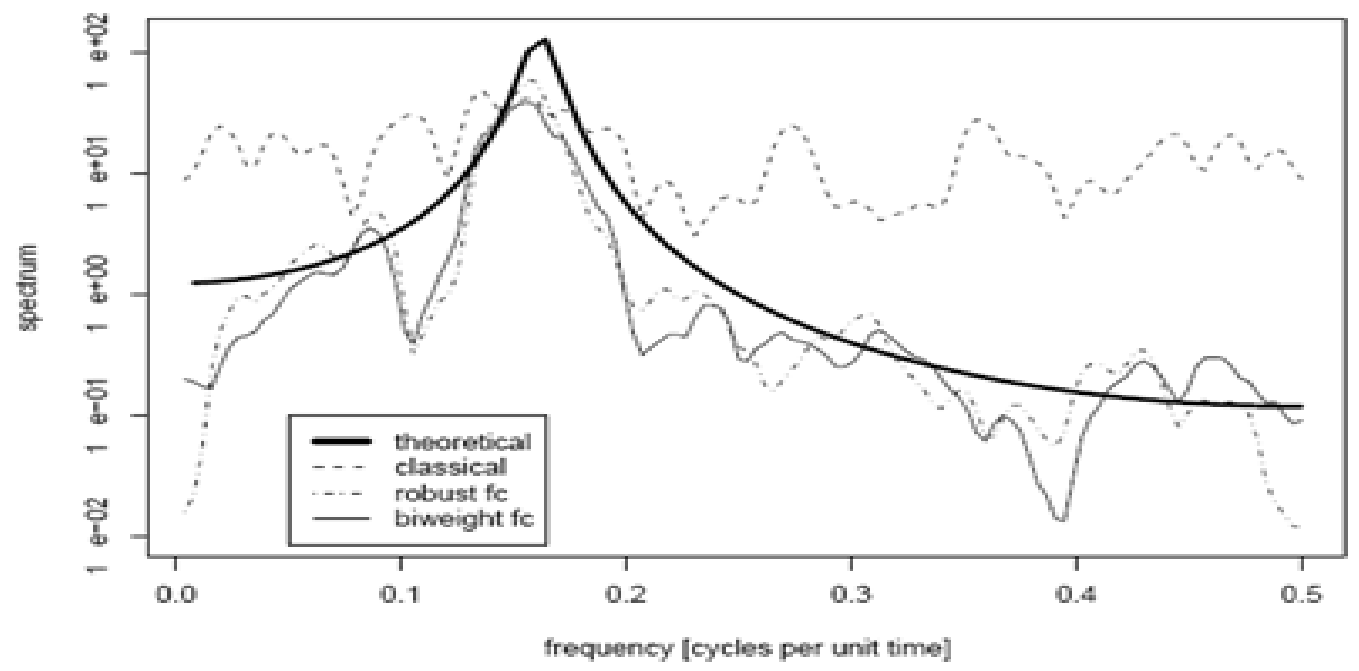

Figure 2: Power spectra estimation in $A R(2)$ model with $10 \%$ AO contamination by robust filter-cleaners: $n=100, M=400$

changed for another and then it returns to the previous state.

Below, the following disorder model is used: $x_{t}=-0.6 x_{t-1}-0.6 x_{t-2}+\epsilon_{t}$ as the main process observed at $t=0,1, \ldots, 400$ and at $t=512, \ldots, 1024 ; x_{t}=x_{t-1}-0.9 x_{t-2}+\epsilon_{t}$ as the disorder process at $t=401, \ldots, 511$. The results of signal processing are exhibited in Figs. 5-6: the classical periodogram indicates two spectrum peaks of the main and contamination processes, whereas the median periodogram indicates only one peak of the main process. 


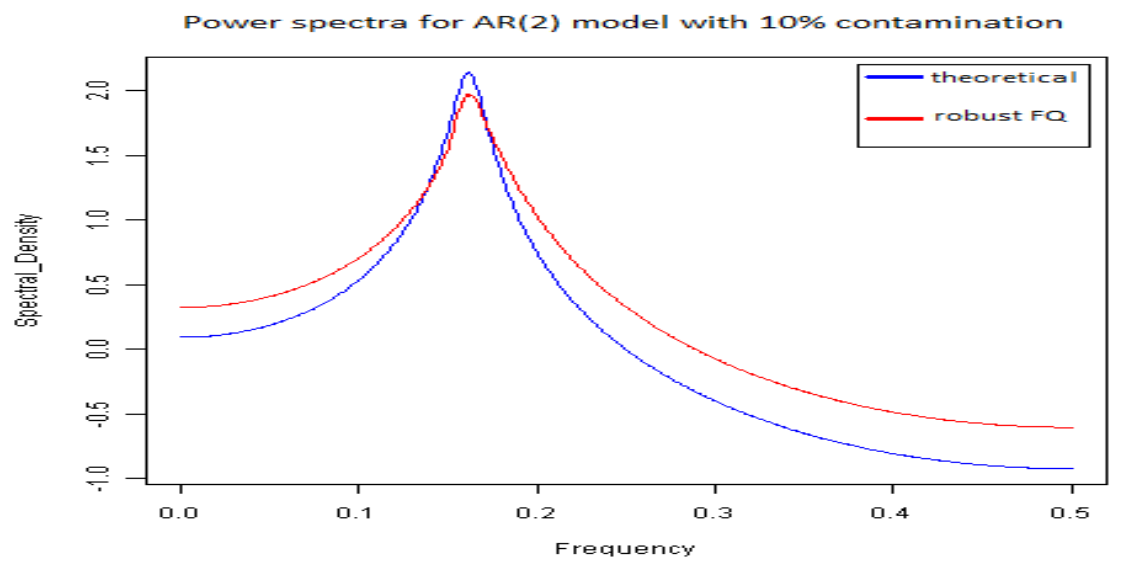

Figure 3: Robust Yule-Walker power spectra estimation in $A R(2)$ model with $10 \%$ AO contamination: $\mathrm{n}=128, \mathrm{M}=2000$

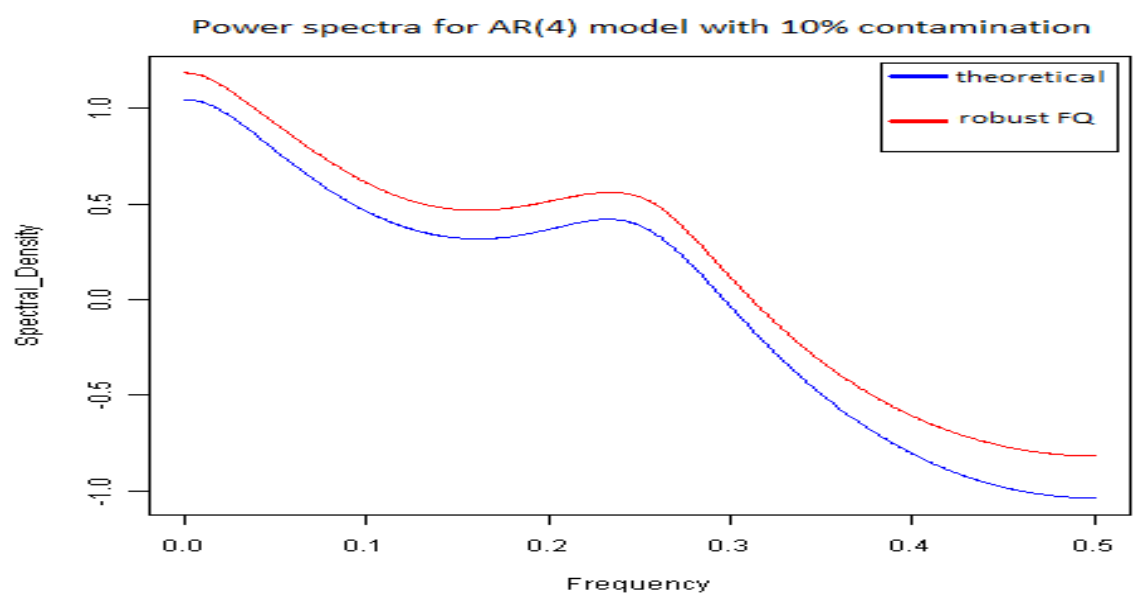

Figure 4: Robust Yule-Walker power spectra estimation in $A R(4)$ model with $10 \%$ AO contamination: $\mathrm{n}=128, \mathrm{M}=2000$

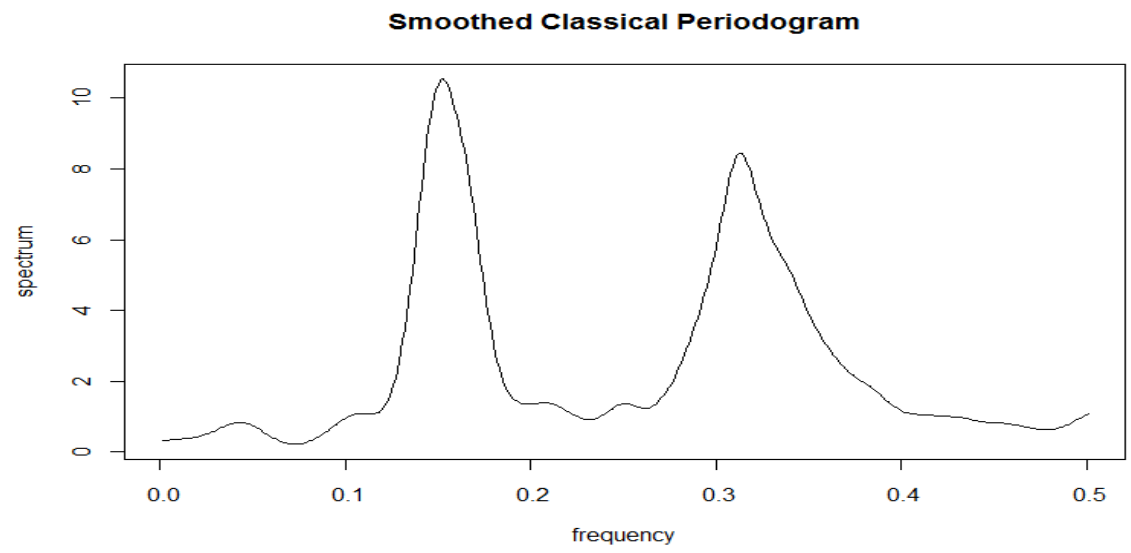

Figure 5: Smoothed classical power spectra estimation in disorder model with $10 \%$ contamination

\section{Concluding remarks}

1) From Figs. 2-3 it follows that the classical periodogram is catastrophically bad under contamination, and that the robust $F Q$ Yule-Walker estimate considerably outperforms robust 


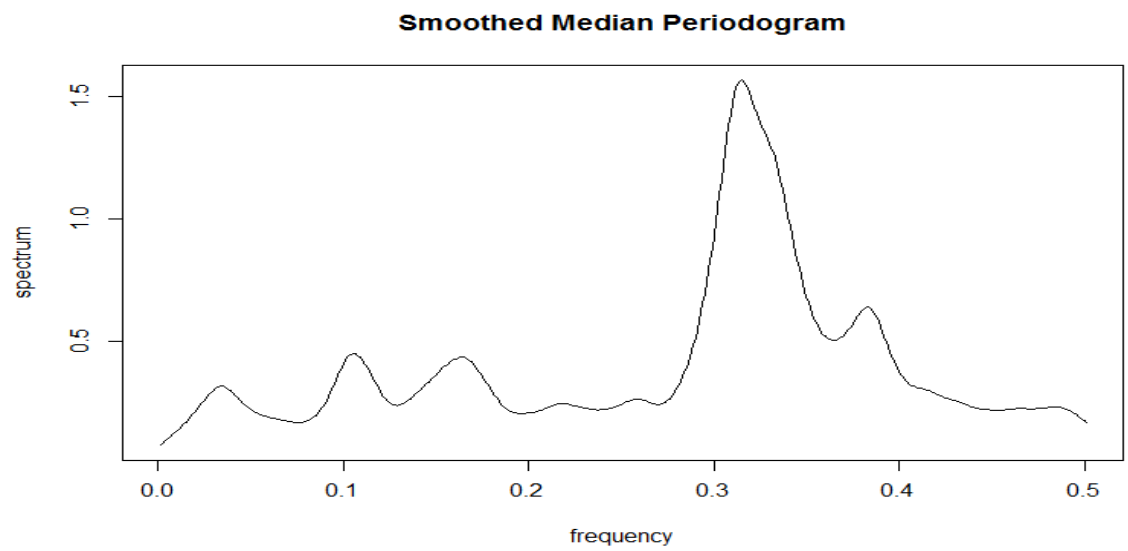

Figure 6: Smoothed robust power spectra estimation in disorder model with $10 \%$ contamination

filter methods.

2) From Fig. 4 it follows that the bias of estimation by the $F Q$ Yule-Walker method increases with growing dimension and contamination. It can be also shown that under heavy contamination, the median periodogram and the robust Blackman-Tukey method outperform the $F Q$ Yule-Walker method in estimating the peak location, although they have a considerable bias in amplitude.

3) The median periodogram exhibits high robustness both with respect to amplitude outliers and to disorder contamination.

4) The obtained results indicate many open problems: analysis of the asymptotic properties of the proposed estimates, reducing their bias and variance on finite samples, and study of the properties of the direct and inverse $L_{p}$-norm analogs of the Fourier transform.

\section{References}

Blackman, R.B., and Tukey, J.W. (1958). The Measurement of Power Spectra. New York: Dover.

Bloofield, P. (1976). Fourier Analysis of Time Series: An Introduction. New York: Wiley.

Brockwell, P.J., and Davis, R.A. (1991). Time Series: Theory and Methods. New York: Springer.

Cover, T.M., and Thomas, J.A. (1991). Elements of Information Theory. New York: Wiley. Hampel, F.R., Ronchetti, E.M., Rousseeuw, P.J., and Stahel, W.A. (1986). Robust Statistics. The Approach Based on Influence Functions. New York: Wiley.

Huber, P.J. (1981). Robust Statistics. New York: Wiley.

Kleiner, B., Martin, R.D., and Thomson, D.J. (1979). Robust Estimation of Power Spectra. Journal of the Royal Statistical Society, B., 41,313-351.

Letac, G. (2011). Does there exist a copula in $n$ dimensions with a given correlation matrix? International Conference on Analytical Methods in Statistics (AMISTAT 2011). October 27-30, Prague, the Czech Republic.

Maronna, R., Martin, D., and Yohai, V. (2006). Robust statistics. Theory and methods. New York: Wiley.

Rousseeuw, P.J., and Croux, C. (1993). Alternatives to the Median Absolute Deviation. Journal of American Statistical Association, 88, 1273-1283.

Pashkevich, M.E., and Shevlyakov, G.L. (1995). The Median Analog of the Fourier Transform. In: Proceedings of the CDAM 1995. Minsk, Belarus. (in Russian) 
Shevlyakov, G.L., Smirnov, P.O., Shin, V.I., and Kim, Kiseon. (2012). Asymptotically Minimax Bias Estimation of the Correlation Coefficient for Bivariate Independent Component Distributions. Journal of the Multivariate Analysis, 111, 59-65.

Shevlyakov, G.L., and Smirnov, P.O. (2011). Robust Estimation of the Correlation Coefficient: An Attempt of Survey. Austrian Journal of Statistics, 40, 147-156.

Smirnov, P.O., and Shevlyakov, G.L. (2010). On Approximation of the $Q_{n}$-Estimate of Scale by Fast $M$-Estimates. In: Book of Astracts of the International Conference on Robust Statistics 2010 (ICORS 2010). Prague, the Czech Republic, pp. 94-95.

Spangl B., and Dutter R. (2005). On Robust Estimation of Power Spectra. Austrian Journal of Statistics, 34, 199-210.

Spangl B. (2008). On Robust Spectral Density Estimation. Dissertation. Technical University of Vienna.

\section{Affiliation:}

Georgy Shevlyakov, Nickolay Lyubomishchenko and Pavel Smirnov

St. Petersburg State Polytechnic University

St. Petersburg, Russia

E-mail: shev@gist.ac.kr

\section{Austrian Journal of Statistics}

published by the Austrian Society of Statistics

Volume 43/3-4

June 2014 http://www.ajs.or.at/

http://www.osg.or.at/

Submitted: 2013-11-25

Accepted: 2014-03-10 